\title{
Philosophiques
}

\section{Pourquoi délibérer ? Du potentiel épistémique à la justification publique}

\section{Marc-Kevin Daoust}

Volume 43, numéro 1, printemps 2016

URI : https://id.erudit.org/iderudit/1036466ar

DOI : https://doi.org/10.7202/1036466ar

Aller au sommaire du numéro

\section{Éditeur(s)}

Société de philosophie du Québec

\section{ISSN}

0316-2923 (imprimé)

1492-1391 (numérique)

Découvrir la revue

\section{Citer cet article}

Daoust, M.-K. (2016). Pourquoi délibérer ? Du potentiel épistémique à la justification publique. Philosophiques, 43(1), 23-48.

https://doi.org/10.7202/1036466ar

\section{Résumé de l'article}

Cet article a deux objectifs. Le premier est de montrer pourquoi l'argument instrumental en faveur de la démocratie est insuffisant pour justifier la délibération politique. Si notre but est l'optimisation du potentiel épistémique d'un régime politique, et que des approches agrégatives et inférentielles (sans délibération) atteignent cet objectif, alors nous ne pouvons plus justifier la délibération sur cette base. Ce problème peut être contourné en reprenant une distinction de Daniel Andler. Pour ce dernier, le groupe délibératif se distingue du groupe agrégatif parce qu'il permet aux agents, par une occasion éducative informelle, de se responsabiliser. La délibération présente donc un potentiel épistémique supérieur, mais qui ne se limite pas à identifier la meilleure réponse dans un temps donné. Le second objectif de l'article est de montrer comme l'argument d'Andler fait le pont entre, d'une part, les théories de la justification publique et, d'autre part, les théories de la démocratie épistémique. 


\title{
Pourquoi délibérer? Du potentiel épistémique à la justification publique MARC-KEVIN DAOUST ${ }^{1}$
}

\author{
Université de Montréal \\ marc-kevin.daoust@umontreal.ca
}

\begin{abstract}
RÉSUMÉ. - Cet article a deux objectifs. Le premier est de montrer pourquoi l'argument instrumental en faveur de la démocratie est insuffisant pour justifier la délibération politique. Si notre but est l'optimisation du potentiel épistémique d'un régime politique, et que des approches agrégatives et inférentielles (sans délibération) atteignent cet objectif, alors nous ne pouvons plus justifier la délibération sur cette base. Ce problème peut être contourné en reprenant une distinction de Daniel Andler. Pour ce dernier, le groupe délibératif se distingue du groupe agrégatif parce qu'il permet aux agents, par une occasion éducative informelle, de se responsabiliser. La délibération présente donc un potentiel épistémique supérieur, mais qui ne se limite pas à identifier la meilleure réponse dans un temps donné. Le second objectif de l'article est de montrer comme l'argument d'Andler fait le pont entre, d'une part, les théories de la justification publique et, d'autre part, les théories de la démocratie épistémique.
\end{abstract}

ABSTRACT - This paper challenges the claim that deliberative democracy is, from an epistemic point of view, an optimal decision-making method. Aggregative and inferential methods can lead collectivities to identify the right or best decisions without having to deliberate. I will argue that this objection can be ruled out if we admit a broader notion of epistemic potential, which would include the informal education and epistemic responsibilities agents gain by deliberating. I will then argue that such a notion of epistemic potential - which includes optimal decisions, epistemic responsibilities and informal education - narrows the gap between theories of epistemic democracy and theories of public justification.

Sur quelle base devrions-nous justifier le régime démocratique, et en particulier, la démocratie délibérative? Il existe des justifications épistémiques, ainsi que des justifications non épistémiques, de la délibération collective. Les justifications épistémiques reposent sur des objectifs tels que la recherche des meilleures solutions à des problèmes donnés, ou encore à l'importance, pour une collectivité, de rechercher la vérité. Pour Landemore et Estlund, l'intérêt de la délibération politique réside dans le fait qu'une telle procédure

1. Une version écourtée de cet article a été présentée au colloque L'expérience de la démocratie: Entre expertise et expérimentation (Rimouski, 26 mai 2015). L'auteur aimerait remercier les évaluateurs ou évaluatrices anonymes, François Claveau, Frédéric Côté-Boudreau, Samuel Dishaw, Jessy Giroux, Jérôme Gosselin-Tapp, Éliot Litalien, Gabriel Monette, Christian Nadeau et Margaux Ruellan pour leurs commentaires judicieux. Cette recherche a été financée par la Chaire de recherche du Canada en épistémologie pratique et le Fonds de recherche du Québec - Société et Culture (FRQSC). 
favorise l'atteinte des meilleures décisions possibles dans un temps donné2. Le potentiel épistémique de la délibération lui conférerait une supériorité face, par exemple, aux décisions prises sans consultation.

Les justifications non épistémiques reposent sur des objectifs tels que l'importance de résoudre les conflits politiques dans le respect des droits de chacun $^{3}$. Par exemple, pour Dilhac, la délibération politique est primordiale parce qu'elle permet l'expression des différences entre les groupes dans le respect d'un certain civisme ${ }^{4}$. La vie commune n'ayant pas pour objectif de trouver la vérité , la délibération collective ne devrait pas être justifiée sur la base du fait qu'elle est un moyen efficace pour atteindre la vérité. De plus, pour Urbinati, les procédures démocratiques comme la délibération sont légitimes parce qu'elles favorisent la liberté et l'égalité citoyenne, notamment par l'inclusion de toutes les personnes concernées dans une procédure décisionnelle commune ${ }^{6}$. Que la délibération s'accompagne de résultats épistémiquement valables est sans doute appréciable, mais un tel constat n'a pas pour effet d'augmenter la légitimité du processus délibératif7?

Si plusieurs prennent position dans le débat entre justification épistémique et justification non épistémique de la délibération démocratique, rares sont les contributions qui interrogent la nature spécifique du potentiel épistémique associé à la délibération. En d'autres termes, on admet qu'il existe une relation entre I) la délibération collective et 2) l'identification de la meilleure solution possible dans un temps donné, et que cette relation

2. David Estlund, «Beyond Fairness and Deliberation: the Epistemic Dimension of Democratic Authority", in James Bohman et William Rheg, dir., Deliberative Democracy: Essays on Reason and Politics, Cambridge, MIT Press, I997, p. I74; Hélène Landemore, «Pourquoi le grand nombre est plus intelligent que le petit nombre, et pourquoi il faut en tenir compte", Philosophiques, vol. 40, $\mathrm{n}^{\circ}$ 2, 2013, p. 284-285.

3. Ce résumé des arguments non épistémiques en faveur de la démocratie délibérative est incomplet. Il existe au moins deux autres justifications non épistémiques de la délibération, que l'on trouve chez les théoriciens du conflit et chez les théoriciens de la négociation. Dans ces perspectives, la délibération est souhaitable parce qu'elle est l'expression d'antagonismes politiques, ou parce qu'elle permet des tractations et des compromis entre les agents d'une collectivité. Pour en savoir davantage sur ces arguments, on peut consulter John P. McCormick, "Machiavelli Against Republicanism - On the Cambridge School's "Guicciardinian Moments" ", Political Theory, vol. 3 I, n ${ }^{\circ}$, 2003, p. 6I 5-643. Mouffe, Chantal, "Le politique et la dynamique des passions ", Politique et sociétés, vol. 22, no 3, 2003, p. I43-I 54. Bernard Manin, "Comment promouvoir la délibération démocratique?", Raisons politiques, vol. 42, $\mathrm{n}^{\circ}$ 2, 20II, p. 83-II3. Jon Elster, "L'usage stratégique de l'argumentation ", Négociations, vol. 2, $\mathrm{n}^{\circ}$ 4, 2005, p. 59-82; et Jane Mansbridge et al., "The Place of Self-Interest and the Role of Power in Deliberative Democracy* ", Journal of Political Philosophy, vol. I8, n I, 2010, p. 64-100.

4. Marc-Antoine Dilhac, La tolérance, un risque pour la démocratie? Théorie d'un impératif politique, Paris, Librairie philosophique J. Vrin, 20I4, p. 49-50.

5. Ibid., p. 57.

6. Nadia Urbinati, Democracy Disfigured, Harvard University Press, 2014, p. I9.

7. Ibid., p. 20,98 . 
exprime le potentiel épistémique spécifique à la délibération. Et si un critère comme «l'atteinte des meilleures décisions dans un temps donné » était une indication inadéquate de ce potentiel épistémique?

Le pari de cet article est double. Non seulement peut-on identifier un critère épistémique qui distingue véritablement la délibération d'autres processus décisionnels, mais l'identification d'un tel critère pourrait permettre un rapprochement entre les justifications épistémique et non épistémique de la délibération.

\section{L'objectif de la meilleure décision, entre agrégation et délibération}

La justification épistémique de la démocratie délibérative, développée notamment par Landemore et Estlund, a fait l'objet de critiques importantes. Dans les dernières années, de nombreux théoriciens ont remis en cause la possibilité d'un progrès épistémique par la délibération. Plutôt que de mener à une décision plus nuancée et à un progrès, la délibération renforce parfois les opinions individuelles ${ }^{8}$, met en valeur les agents charismatiques ${ }^{9}$, génère de la pensée de groupe $^{10}$, écarte certains groupes économiques ou raciaux de la discussion collective ${ }^{11}$ ou cause des blocages institutionnels ${ }^{12}$. Tous ces éléments sont des obstacles au fait d'identifier la meilleure décision dans un temps donné.

Il y a au moins deux façons de réagir à ces problèmes: d'une part, nous pourrions considérer que cette critique est très pertinente pour réformer la délibération, en vue de l'améliorer. D'autre part, nous pourrions considérer que cette critique est concluante pour abandonner le modèle délibératif, et ce, au profit d'un modèle agrégatif ou inférentiel. Le potentiel épistémique des modèles agrégatifs est utilisé, par exemple, pour l'achat en ligne. Lorsqu'une personne fait des achats en ligne, un algorithme collecte et classe la toile de ses préférences, et utilise cette toile pour suggérer des achats aux autres utilisateurs. Plus le modèle dispose d'informations sur les différents utilisateurs, plus il est apte à prédire rapidement les préférences des utilisateurs futurs. Ainsi, à long terme, le modèle peut prédire quelles seraient les meilleures décisions d'achats pour tous les types d'utilisateurs ${ }^{13}$, et cette pré-

8. Cass R Sunstein, Infotopia: How Many Minds Produce Knowledge, Oxford University Press, 2006, p. 45, ibid.

9. Lynn M. Sanders, "Against Deliberation", Political Theory, vol. 25, n 3, I997, p. 347, Surowiecki, The Wisdom of Crowds, p. I82.

10. Ibid., chap. II, $\mathbb{S}$, Miriam Solomon, "Groupthink versus The Wisdom of Crowds: The Social Epistemology of Deliberation and Dissent ", The Southern Journal of Philosophy, vol. $44, \mathrm{n}^{\circ}$ SI, 2006, p. 28.

11. Sanders, "Against Deliberation", p. 353.

12. Mark Klein, «How to Harvest Collective Wisdom on Complex Problems: An Introduction to the MIT Deliberatorium », $20 \mathrm{I} 2$.

13. Bien sûr, le potentiel épistémique est parfois réduit dans le cas d'objectifs marchands, que ce soit pour favoriser certains produits ou augmenter les recettes publicitaires. Il n'en demeure pas moins que le modèle pourrait être libre de ces biais marchands et offrir un potentiel épistémique maximal. 
diction est le résultat d'un processus faisant l'économie de la délibération entre les utilisateurs.

Si les modèles agrégatifs présentent un bon potentiel épistémique, pour quelles raisons devrions-nous défendre la délibération plutôt que l'agrégation? Bien que les modèles agrégatifs connaissent un grand succès dans ce que nous appelons les théories de la sagesse collective (collective wisdom), ils suscitent nombre de questions. Les approches anti-délibératives supposent que la délibération n'est pas une étape nécessaire ni suffisante à l'intelligence collective. Dans certaines situations, il vaudrait donc mieux ne pas délibérer. Plutôt que de délibérer, on procède à l'agrégation des préférences, avec des mécanismes ou des algorithmes. Il suffit qu'un groupe présente une diversité suffisante ${ }^{14}$, ainsi qu'un bon degré de sophistication ${ }^{15}$, pour qu'une agrégation des préférences et des informations permette au groupe de prendre une très bonne décision.

Supposons que l'agrégation, d'un point de vue épistémique, soit parfois supérieure. La question que l'on peut alors se poser est la suivante: pourrions-nous remplacer les cadres délibératifs politiques par des algorithmes d'agrégation des préférences? À première vue, un modèle agrégatif et inférentiel répondrait aux «attentes épistémiques» que nous avons à l'égard de la délibération. Juliette Roussin résume cette idée de la manière suivante:

Le collectif est en quelque sorte délivré des pesanteurs de la théorie délibérative: [...] un groupe peut produire de sages décisions et prédictions par simple agrégation, sans avoir à se plier aux rituels contraignants, parfois irréalistes, prescrits par la théorie - tels que la reconnaissance sincère par les partenaires de normes de rationalité partagées devant idéalement permettre, au terme d'une discussion réglée, d'aboutir à un consensus rationnel autour du meilleur $\operatorname{argumen} t^{16}$.

\section{Le plan de cet article}

Après un rappel succinct de «l'argument instrumental» de Landemore et Estlund en faveur de la démocratie (sect. I), nous montrerons les limites d'une telle thèse, notamment en ce qui concerne la pensée de groupe, les rapports de domination ou la polarisation. Nous montrerons ensuite que les modèles agrégatifs ne présentent pas ces problèmes, et exposerons les principaux arguments développés par Page, Hong, Servan-Schreiber et Vermeule

14. Scott E. Page, The Difference: How the Power of Diversity Creates Better Groups, Firms, Schools, and Societies, Princeton (NJ), Princeton University Press, 2007.

15. Lu Hong et Scott Page, "The Micro-Foundations of Collective Wisdom ", in Jon Elster et Hélène Landemore, dir., Collective Wisdom: Principles and Mechanisms, Cambridge, Cambridge University Press, 20I2, p. 56-7I.

16. Juliette Roussin, «Sagesse des foules », La vie des idées, 2I septembre 20I 2, [http:// www.laviedesidees.fr/Sagesse-des-foules.html], sect. 5 . 
en faveur d'un modèle agrégatif (sect. 2). Nous proposerons quelques nuances à ce modèle, notamment par les contributions de Gloria Origgi et Christian List, mais ces nuances seront insuffisantes pour rejeter le modèle agrégatif. Nous présenterons ensuite l'argument d'Andler, selon lequel il ne faut surtout pas confondre sagesse et intelligence collective. Si la délibération collective permet la sagesse, l'agrégation ne permet que l'intelligence collective (sect. 3 ).

Au terme de l'argument d'Andler, nous admettrons une conception plus large du potentiel épistémique, qui inclut l'éducation informelle des acteurs à travers la discussion collective. L'argument instrumental pourrait alors être réformé pour inclure l'ensemble de ce potentiel épistémique. Cela dit, une telle conception du potentiel épistémique pourrait nous laisser croire que l'enjeu «éducationnel » dont fait état Andler s'assimile à une exigence de justification publique, et dont les effets épistémiques sont collatéraux. En d'autres termes, nous pourrions croire qu'un cadre éducatif informel pourrait être justifié sur la seule base du besoin, en démocratie, que les citoyens comprennent bien les décisions collectives.

Nous montrerons toutefois que l'éducation informelle et l'impératif de justification publique sont indissociables (sect. 4). Plus particulièrement, accepter l'idée de justification publique, c'est nécessairement favoriser les cadres qui ont le meilleur potentiel épistémique. L'argument d'Andler a donc deux effets majeurs. D'une part, accepter cet argument revient à modifier, de façon substantielle, ce que nous qualifions «d'argument instrumental» en faveur de la démocratie. D'autre part, l'argument d'Andler opère un rapprochement important entre les théories de la démocratie épistémique et l'exigence de justification publique des décisions communes.

Au final, le but de cet article est de mieux comprendre le rôle de la délibération dans les théories épistémiques de la démocratie, et de mieux comprendre la relation entre potentiel épistémique et exercice délibératif. La démocratie épistémique est couramment critiquée sur plusieurs aspects. Par exemple, invoquer le potentiel épistémique de la démocratie serait au minimum superflu, par rapport à ce qui constituerait les «vraies » justifications de la démocratie pour ce qui est de la légitimité et de la participation ${ }^{17}$. Des craintes persistent aussi quant au lien entre démocratie épistémique et régime des experts ${ }^{18}$. Pourtant, comme le montrera cet article, si on procède à l'examen critique et détaillé de la notion de potentiel épistémique au sein de la délibération, la démocratie épistémique apparaît alors comme un élément incontournable à l'exercice de la justification publique.

17. Urbinati, Democracy Disfigured, chap. II.

18. Pour une bonne analyse de ces critiques et des solutions à ce problème, on peut se référer à Jessy Giroux, «Le spectre épistocratique ", Philosophiques, vol. 40, $\mathrm{n}^{\circ}$ 2, 20I3, p. 3OI-3I9. 


\section{La délibération et l'argument instrumental en faveur de la démocratie}

L'argument instrumental en faveur de la démocratie suppose d'abord que la valeur épistémique d'une procédure influence en partie la légitimité d'une décision. Comme l'indique Estlund dans le passage suivant, "the legitimacy of an outcome depends [...], partly, from the epistemic value, even though it is imperfect, of the procedure that produced $i t^{19}$ ". Si une procédure décisionnelle génère des gains épistémiques, alors, ceteris paribus, la décision qui en émane devrait être porteuse d'une plus grande légitimité. C'est dans cette optique, axée sur les gains épistémiques, que l'argument instrumental en faveur de la démocratie préconise une approche délibérative quant aux décisions politiques ${ }^{20}$.

La délibération est une méthode décisionnelle centrée sur les échanges entre participants ${ }^{21}$. Ces échanges se veulent rationnels en vue de prendre la meilleure décision possible. La diversité et la quantité d'agents participant librement à la délibération nous permettent d'exprimer de nombreux points de vue. En d'autres termes, si tous les agents ont un profil socio-économique, académique ou culturel identique, alors l'analyse du problème et des méthodes employées pour le résoudre risque d'être limitée.

Le potentiel épistémique de la délibération lui confère une supériorité face, par exemple, aux décisions prises sans consultation. Les échanges entre des acteurs diversifiés mènent progressivement à modifier les points de vue exprimés. "Deliberation is supposed to 1) enlarge the pool of ideas and information; 2) weed out the good arguments from the bad; and 3) lead to a consensus on the "best" or more "reasonable" solution ${ }^{22}$." Théoriquement, la délibération atteint donc ses objectifs lorsqu'un consensus apparaît autour d'une position ou une action ${ }^{23}$. Le potentiel épistémique de la démocratie délibérative est supérieur parce qu'une idée adoptée démocratiquement a fait l'objet d'un examen collectif ${ }^{24}$.

L'examen collectif d'une thèse est mené par une collectivité diversifiée, où tous les statuts socio-économiques, culturels et académiques sont pris en considération. La diversité des agents est centrale, puisqu'elle permet

19. Estlund, «Beyond Fairness and Deliberation", p. I74.

20. Urbinati, Democracy Disfigured, p. 93-94; Alice Le Goff et Christian Nadeau, "Autorité démocratique et contestation. L'apport d'une approche épistémique», Philosophiques, vol. 40, $\mathrm{n}^{\circ}$ 2, $20 \mathrm{I} 3$, p. 257.

21. Pour une étude minutieuse des différentes définitions de la délibération, on peut consulter Manin ( "Comment promouvoir la délibération démocratique? ", p. IOI-IO8).

22. Hélène E. Landemore, «Democratic Reason: The Mechanisms of Collective Intelligence in Politics ", in Hélène Landemore et Jon Elster, dir., Collective Wisdom: Principles and Mechanisms, Cambridge, Cambridge University Press, 20I2, p. 257.

23. Ibid., p. 254-56.

24. Jessy Giroux, "Le spectre épistocratique", Philosophiques, vol. 40, $\mathrm{n}^{\circ}$ 2, 2013, p. 3I4-3I5; Le Goff et Nadeau, "Autorité démocratique et contestation. L'apport d'une approche épistémique ", p. 263. 
d'élargir le spectre des idées, des heuristiques et des faits, ainsi que de mener la critique la plus complète des idées reçues. Or, dans les régimes autoritaires ou fortement hiérarchisés, on sape la diversité des points de vue. Dans ces régimes, les décisions sont prises par un petit groupe d'individus peu diversifié. Même si ces individus décident de consulter une collectivité pour prendre la meilleure décision possible, ce choix s'avère contingent, puisque dans les faits, ils pourraient prendre une décision sans consultation ${ }^{25}$.

Landemore tire du potentiel épistémique de la délibération collective ce que nous pourrions qualifier d' "argument instrumental» en faveur de la démocratie. L'argument de Landemore peut être présenté ainsi: un régime politique devrait favoriser les mécanismes décisionnels ayant une valeur épistémique supérieure. La démocratie délibérative inclut, de par sa nature même, des mécanismes décisionnels ayant un potentiel épistémique supérieur aux régimes autoritaires ou dictatoriaux ${ }^{26}$. Par conséquent, la démocratie délibérative est préférable à d'autres régimes ${ }^{27}$. Landemore résume sa thèse ainsi: "Combining the epistemic properties of deliberation and majority rule, I conclude that democracy - in theory - is superior to any version of the rule of the few, including when we make unrealistic assumptions about the intelligence of the few ${ }^{28}$.»

\subsection{La délibération et les relations sociales néfastes qu'elle génère}

Une critique que nous pourrions adresser à Landemore et Estlund est qu'ils ne répondent pas aux problèmes sociaux de la délibération. Les acteurs engagés dans une délibération adoptent très souvent des comportements ou des attitudes qui sont néfastes d'un point de vue épistémique. L'observation selon laquelle la délibération ne produit pas les effets désirés est récurrente dans de nombreuses études. Plutôt que de mener à une décision plus nuancée et à un progrès des thèses étudiées, la délibération semble renforcer les convictions individuelles. En d'autres termes, plutôt que de faire preuve d'ouverture, les agents confirmeraient et renforceraient leurs opinions au contact des autres.

L'expérience délibérative analysée par Reid Hastie, David Schkade, et Cass R. Sunstein constitue une illustration emblématique de ce phénomène ${ }^{29}$. Dans une expérience sociale menée au Colorado, à l'été 2005 , on a

25. Frédéric Chanu, "Intelligence collective et démocratie en Politique, III, I I ", Ithaque, $\mathrm{n}^{\circ} \mathrm{I}_{5}, 2014$, p. $80-82$.

26. Juliette Roussin, «Démocratie contestataire ou contestation de la démocratie? L’impératif de la bonne décision et ses ambiguités", Philosophiques, vol. 40, $\mathrm{n}^{\circ}$ 2, 2013, p. 374, 376.

27. Landemore, «Democratic Reason", p. 25 I-253.

28. Ibid., p. 282.

29. Sunstein, Infotopia, chap. II, David Schkade, Cass R. Sunstein et Reid Hastie, "What Happened on Deliberation Day? », ID 9I I 646, Rochester, NY, Social Science Research Network, 2006, [http://dx.doi.org/I0.2I39/ssrn.9I I 646]. 
constitué de petits groupes de discussion avec des personnes généralement conservatrices ou libérales, et on a invité ces personnes à discuter ensemble sur des questions controversées (wedge issues) comme les protocoles internationaux contre les changements climatiques, le mariage homosexuel ou la discrimination positive. Avant de commencer l'expérience, on a mesuré l'opinion des membres de chaque groupe à l'égard de ces questions, sans oublier la force (très convaincu, faiblement convaincu, neutre, etc.) de ces opinions. L'objectif de cette expérience est de mesurer l'impact d'une délibération sur la modification des opinions individuelles. Au terme de l'expérience, le seul résultat significatif était le renforcement des opinions respectives des participants. "The results were simple. In almost every group, members ended up with more extreme positions after they spoke with one another ${ }^{30}$.»

D'autres relations sociales néfastes peuvent aussi prendre racine dans une délibération. On peut penser, par exemple, à la domination rhétorique des agents charismatiques, au fait que certains groupes économiques ou raciaux sont souvent écartés de la discussion collective, ou que certains individus profitent des règles délibératives pour causer des blocages institutionnels (comme le filibusting, par exemple) ${ }^{31}$.

Jusqu'ici, nous n'avons pas montré qu'il fallait rejeter la délibération. La délibération permet parfois de dissiper de fausses croyances ou d'exposer certains présupposés. "A nation's leaders often try to convince citizens that the economy is improving, but deliberation might reveal that the economy is actually getting worse. If some group members have anchored on a misleading value, perhaps deliberation will expose the anchor as such ${ }^{32}$." Seulement, on ne peut nier que l'action délibérative, soit le fait de réunir un groupe qui échange selon une procédure publique, est un phénomène social qui comporte son lot d'obstacles épistémiques. C'est comme phénomène social que la délibération n'échappe pas à certains pressions, distractions ou pièges sociaux comme les biais cognitifs, la pensée de groupe ou le renforcement des positions respectives.

Il existe, bien sûr, des méthodes pour corriger les pressions exercées sur la délibération. Nous pourrions donc corriger les lacunes sociales de cette dernière, à condition de réformer en profondeur notre conception d'une délibération saine et des conditions dans lesquelles elle prend place ${ }^{33}$. Par contre, si un modèle présentait des propriétés épistémiques similaires ou supérieures à la délibération, tout en évitant les écueils ou les coûts liés à

30. Sunstein, Infotopia, p. 45 .

31. Sanders, "Against Deliberation", Solomon, "Groupthink versus The Wisdom of Crowds".

32. Sunstein, Infotopia, p. 48.

33. Ibid., chap. VI. 
cette dernière, alors la nécessité d'avoir recours à la délibération dans l'argument instrumental en faveur de la démocratie serait caduque.

\section{Les approches agrégatives et leur supériorité épistémique}

Plutôt que d'opter pour l'échange entre différents participants, les modèles agrégatifs et inférentiels ${ }^{34}$ assemblent des informations en vue de prendre une décision. Ces modèles peuvent faire usage de la somme des choix, interactions ou données comportementales des agents. Les données sont triées et systématisées pour en extraire des informations valables. On peut penser, par exemple, à un site d'achats en ligne. Imaginons qu'un site d'achats en ligne enregistre l'ensemble de vos données de navigation, y compris l'ordre, le lieu et le temps de consultation des différentes pages. Un tel site peut utiliser ses données et les systématiser grâce à un algorithme. Cet algorithme peut ensuite suggérer des objets à acheter aux autres utilisateurs qui ont un portrait similaire. Plus l'algorithme dispose de données comportementales et socio-économiques au sujet de l'utilisateur, plus il peut faire des suggestions probantes aux autres utilisateurs.

Le modèle n'est pas limité à l'identification des préférences, mais peut aussi s'appliquer à l'identification des informations pertinentes. Prenons le cas des encyclopédies collaboratives. Les utilisateurs sont invités à ajouter leurs connaissances aux différentes entrées d'une encyclopédie ${ }^{35}$. Sans être des experts, ces personnes disposent de connaissances justes à propos d'un objet. Or, puisque des millions d'utilisateurs issus de milieux diversifiés participent à l'encyclopédie, les entrées sont souvent plus complètes et aussi fiables que celles rédigées par des experts. De plus, même s'ils ne sont pas des experts, les individus peuvent repérer des informations incomplètes et les préciser. Comme le degré d'interaction et de diversité est beaucoup plus grand qu'au sein d'un groupe d'experts, un tel modèle réduit les biais systématiques au minimum. Surowiecki résume cette thèse de la manière suivante: "[W]hen our imperfect judgments are aggregated in the right way, our collective intelligence is often excellent ${ }^{36}$."

C'est précisément grâce à leur potentiel épistémique supérieur que se démarquent les modèles agrégatifs. Le fait qu'une foule d'utilisateurs mettent leurs informations en commun permet à l'algorithme d'identifier à coup sûr le bon résultat (outcome) dans un cas particulier. Par exemple, parmi les milliards de pages disponibles sur le web, un moteur de recherche peut, en fonction de mots clés, identifier rapidement la page qu'un utilisateur recherche. Ainsi, le modèle est dit intelligent, parce qu'il offre aux utilisa-

34. Pour simplifier la lecture, on fera désormais référence aux modèles inférentiels et agrégatifs par l'expression «modèle agrégatif».

35. Certaines encyclopédies collaboratives incluent des dimensions délibératives, mais ce n'est pas nécessairement le cas.

36. Surowiecki, The Wisdom of Crowds, p. XIV. 
teurs ce qu'ils recherchent dans des délais très courts. De plus, nous pouvons constater que, contrairement aux modèles délibératifs, les agents ne sont pas obligés de délibérer pour extraire l'information la plus précise possible. Il suffit qu'un algorithme ou un cadre collaboratif permette la mise en commun des informations, et que les utilisateurs présentent un certain degré de sophistication et de diversité, pour que le modèle obtienne un potentiel épistémique égal ou supérieur à la délibération classique.

L'agrégation peut se faire de plusieurs manières. Dans le modèle de Klein, par exemple, l'agrégation se fait par "l'addition stricte» de tous les points de vue sur une question ${ }^{37}$. Imaginons que nous demandons à des participants et participantes de rédiger une entrée encyclopédique sur l'exploitation du pétrole. Plutôt que de débattre sur le contenu de cette entrée, chaque personne serait libre d'y ajouter de l'information. La contestation et la délibération touchant les informations sont substituées par l'ajout d'informations nuancées. Imaginons qu'un écologiste et un actionnaire d'une compagnie pétrolière participent à cette encyclopédie. Plutôt que d'argumenter, chacun alimentera une section de l'encyclopédie. L'écologiste pourra ajouter des éléments sous la rubrique «impact de l'exploitation pétrolière sur les rivières", alors que l'actionnaire pourra ajouter des éléments sous la rubrique «création d'emplois ». Les résultats de l'expérience de Klein montrent qu'une encyclopédie complète, fiable et très précise peut émerger d'une démarche exempte de toute délibération ou contestation.

Le modèle prédictif de Servan-Schreiber est un autre exemple du même ordre, mais appliqué aux transactions sur un marché. Plutôt que de confier l'achat des actions à un spécialiste du marché, imaginons que les agents font leurs transactions directement les uns contre les autres. Prenons un marché prédictif où le produit sur lequel les agents misent est l'occurrence d'un événement (un pari sportif, par exemple). Si les agents achètent des parts d'un stock, c'est qu'ils considèrent que l'événement va se produire. S'ils vendent, c'est qu'ils considèrent que l'événement ne se produira pas. Si le nombre d'utilisateurs est élevé et diversifié, le prix du marché sera des plus fiables. La corrélation observée entre la fiabilité des prédictions et les résultats observés est très forte ${ }^{38}$ : les résultats obtenus sont plus fiables que les évaluations expertes, les sondages d'opinion ou les analyses institutionnelles ${ }^{39}$.

37. Klein, «How to Harvest Collective Wisdom on Complex Problems: An Introduction to the MIT Deliberatorium».

38. Emile Servan-Schreiber, "Trading Uncertainty for Collective Wisdom», in Hélène Landemore et Jon Elster, dir., Collective Wisdom: Principles and Mechanisms, Cambridge, Cambridge University Press, 20I 2, p. 27.

39. Ibid., p. 27-29. 


\subsection{La généralisation du modèle agrégatif: le théorème de Page}

Au-delà de certains résultats empiriques particuliers, nous pourrions douter de la validité générale de telles inférences entre agrégation des préférences et intelligence collective. S'il existe des illustrations empiriques du fait que certaines agrégations s'accompagnent d'un bon potentiel épistémique, il pourrait simplement s'agir d'un hasard. En d'autres termes, ce potentiel pourrait ne pas trouver ses racines dans la nature même de la méthode agrégative.

Pour dissiper ce doute, Scott Page propose un théorème simple de la valeur épistémique des foules ${ }^{40}$. Le "diversity prediction theorem ${ }^{41}$ " stipule que le carré de l'erreur d'une prédiction collective est égal au carré des erreurs moyennes à laquelle on soustrait la "diversité prédictive ${ }^{42}$ ». C'est la diversité prédictive, soit le fait que plusieurs agents font différentes prédictions, qui réduit le carré de l'erreur d'une prédiction collective. De ce premier théorème, on peut conclure que le carré de l'erreur pour une prédiction collective est inférieur ou égal au carré des erreurs moyennes des prédictions individuelles. Sans entrer dans les détails mathématiques de cette preuve, nous pouvons affirmer qu'en général, la foule fait mieux que la moyenne des individus composant le groupe ${ }^{43}$.

Un exemple permet de mieux comprendre le théorème de Page. Supposons que David, Anouk et Camille fassent des prédictions électorales ${ }^{44}$. Ils tentent de prédire combien de circonscriptions remportera chaque parti. Le tableau suivant résume leurs prédictions respectives, ainsi que le résultat réel des élections:

Tableau 1: Prédictions, par participant et participante, du nombre de circonscriptions remportées par chaque parti

\begin{tabular}{|l|c|c|c|}
\hline & Parti rouge & Parti bleu & Parti orange \\
\hline David & I I & I9 & 23 \\
\hline Anouk & I 5 & I7 & 2 I \\
\hline Camille & I6 & 2I & I6 \\
\hline Prédiction collective & I4 & I9 & 20 \\
\hline Résultat réel & I6 & I7 & 20 \\
\hline
\end{tabular}

40. L'expression «foule» renvoie aux groupes ne disposant pas de mécanismes délibératifs. On peut penser au public lors d'un concert, aux participants d'une manifestation, aux différents utilisateurs d'un site web, etc.

41. Scott E. Page, Diversity and Complexity, Princeton (NJ), Princeton University Press, 2010, p. 227.

42. Hong et Page, "The Micro-Foundations of Collective Wisdom », p. 60-6I.

43. Voir Page, The Difference, chap. VIII pour les détails entourant ce résultat.

44. Cet exemple est une adaptation d'une mise en situation que l'on trouve dans Page, The Difference, p. 205-209. 
Avec ces données, on peut calculer le carré de l'erreur pour chaque prédiction individuelle. Par exemple, David a sous-estimé le parti rouge de 5 circonscriptions, a surestimé le parti bleu de 2 circonscriptions, et a surestimé le parti orange de 3 circonscriptions. Le carré de l'erreur moyen pour David serait donc $\left(5^{2}+2^{2}+3^{2}\right) / 3=\mathrm{I} 2,67$. Le tableau suivant résume le carré de l'erreur moyen de chaque participant et participante, ainsi que le carré de l'erreur moyen pour la prédiction collective:

Tableau 2: Carré de l'erreur de chacune des prédictions

\begin{tabular}{|l|l|l|l|l|}
\hline & Parti rouge & Parti bleu & Parti orange & Moyenne \\
\hline David & $(\mathrm{II}-\mathrm{I} 6)^{2}=25$ & $(\mathrm{I} 9-\mathrm{I} 7)^{2}=4$ & $(23-20)^{2}=9$ & $\mathrm{I} 2,67$ \\
\hline Anouk & $(\mathrm{I} 5-\mathrm{I} 6)^{2}=\mathrm{I}$ & $(\mathrm{I} 7-\mathrm{I} 7)^{2}=0$ & $(2 \mathrm{I}-20)^{2}=\mathrm{I}$ & 0,67 \\
\hline Camille & $(\mathrm{I} 6-\mathrm{I} 6)^{2}=0$ & $(\text { II-I7 })^{2}=\mathrm{I} 6$ & $(\mathrm{I} 6-20)^{2}=\mathrm{I} 6$ & I0,67 \\
\hline Prédiction collective & $(\mathrm{I} 4-\mathrm{I} 6)^{2}=4$ & $(\mathrm{I} 9-\mathrm{I} 7)^{2}=4$ & $(20-20)^{2}=0$ & 2,67 \\
\hline
\end{tabular}

Si on fait la moyenne du carré de l'erreur de chaque prédiction individuelle, on obtient $(\mathrm{I} 2,67+0,67+\mathrm{I} 0,67) / 3=8$. En d'autres termes, les différents individus composant le groupe ont, en moyenne, un carré de l'erreur de 8 pour chacune de leurs prédictions. Or la moyenne des carrés de l'erreur de la prédiction collective est de 2,67 , soit 3 fois moins que pour les prédictions prises individuellement. Bien que certains agents (comme Anouk) s'en tireraient peut-être mieux s'ils préféraient leur prédiction à la prédiction collective, il reste que, pour la majorité des membres composant le groupe, la prédiction collective réduit davantage l'erreur que leurs propres prédictions individuelles.

L'intérêt du théorème de Page est de montrer qu'il y a un fondement probabiliste à la réduction de l'erreur par l'agrégation. De plus, la réduction de l'erreur est proportionnelle à la diversité et à la quantité d'utilisateurs qui participent au modèle agrégatif. Dans un modèle alimenté par des millions d'utilisateurs diversifiés, le taux d'erreur tend à être résiduel. Au contraire, pour des raisons d'espace ou de participation égale à la discussion, la délibération ne peut pas faire interagir des millions de participants. En ce sens, l'agrégation réduit le degré d'erreur plus aisément que la délibération.

Pour que le résultat de Page soit valide, le groupe doit toutefois présenter certaines caractéristiques. Ce qui assure que l'erreur est plus faible est le degré de sophistication et de diversité des membres composant le groupe ${ }^{45}$. Les individus issus de milieux distincts n'enrichissent pas le modèle de la même manière ${ }^{46}$. Si l'on peut compter sur une bonne diversité, alors le

45. Hong et Page, "The Micro-Foundations of Collective Wisdom", p. 65.

46. Ibid., p. 57. 
modèle sera alimenté par des sources et des éléments très diversifiés, ce qui contribue à parfaire le modèle. Ainsi, les individus au sein du groupe doivent présenter différents portraits socio-économiques, et doivent suivre des méthodes (models) différentes ${ }^{47}$. Enfin, les individus doivent aussi présenter des connaissances minimales de l'objet analysé ${ }^{48}$.

Revenons aux traits distinctifs de l'agrégation par rapport à la délibération, et à la façon dont cela affecte l'argument instrumental de Landemore et Estlund. Si c'est vraiment la recherche d'un potentiel épistémique qui nous pousse à adopter ou non le régime démocratique, alors nous pourrions très bien justifier l'abandon de la délibération commune au profit de l'agrégation commune des préférences ${ }^{49}$. Plutôt que de favoriser la délibération, nous pourrions favoriser la diversité et la sophistication au sein du groupe. Ainsi, le problème de l'argument instrumental, c'est que nous pouvons abandonner la délibération au profit de l'agrégation, sans pour autant que cela n'affecte le cœur de l'argument. Adrian Vermeule résume cette idée d'une manière très claire dans le passage suivant:

Deliberation is also unnecessary for the operation of perspectival aggregation. Consider Aristotle's idea of the "Wisdom of the Multitude" [...]. Altough some theorists seem to assume that his idea has a necessary connection to deliberation, it does not. Perspectival aggregation can be accomplished just by having each participant unilaterally reveal a bit of dispersed information to the group, without deliberation ${ }^{50}$.

\subsection{Des doutes quant à l'abandon de la délibération: Origgi et l'approche par la réputation}

Certains auteurs remettent néanmoins en cause la possibilité d'abandonner la délibération au profit de la stricte agrégation. C'est le cas du modèle basé sur la réputation d'Origgi. Pour Origgi, l'information produite par les moteurs de recherche ou les agrégateurs de contenu semble fiable, mais une méthode indépendante doit exister pour conférer une valeur à ces algorithmes $^{51}$. Par exemple, comment un moteur de recherche peut-il affirmer qu'il répond aux besoins de ses utilisateurs s'il n'existe aucune source indépendante témoignant de la satisfaction des utilisateurs? Des mécanismes de

47. Il n'y a pas d'opposition entre ces deux caractéristiques des groupes. "Crowd members can become both more sophisticated and more diverse" (ibid.)

48. Surowiecki, The Wisdom of Crowds, p. I83.

49. Adrian Vermeule, "Collective Wisdom and Institutional Design ", in Hélène Landemore et Jon Elster, dir., Collective Wisdom: Principles and Mechanisms, Cambridge, Cambridge University Press, 20I 2, p. 347-348.

50. Ibid., p. 348.

51. Gloria Origgi, «Designing Wisdom through the Web: Reputation and the Passion for Ranking ", in Hélène Landemore et Jon Elster, dir., Collective Wisdom: Principles and Mechanisms, Cambridge, Cambridge University Press, 20I 2, p. 39. 
tri ou des filtres doivent permettre d'évaluer la qualité des agrégations. Les formes possibles de l'évaluation sont multiples: les classements, la vérification par les pairs ou le fait que des groupes posent un second regard sur le produit de l'agrégation sont autant de façons d'évaluer l'agrégation. Il y a donc intelligence collective si des agrégations de données sont faites par l'intermédiaire des nouvelles technologies, mais surtout si cette agrégation fait l'objet d'un examen critique par des pairs ${ }^{52}$. Or un examen critique fait appel à des interventions plus substantielles que le simple fait d'indiquer quelles sont nos préférences.

La réputation peut se mesurer par des mécanismes comme la notation ou l'ordination (le fait de conférer un rang à un modèle $)^{53}$. Les participants peuvent ensuite décider individuellement s'ils acceptent ou non le produit de l'agrégation, selon qu'ils jugent que la réputation de l'agrégateur est bonne ou mauvaise. L'évaluation collective de la réputation d'un algorithme est nécessaire, notamment parce qu'elle est le seul mécanisme de contestation ou de remise en cause de l'algorithme. En d'autres termes, ce serait le seul moyen de vérifier a posteriori le bon fonctionnement de l'algorithme. Ainsi, l'agrégation ne saurait entièrement remplacer la délibération, puisqu'au moins une délibération a posteriori touchant la construction de l'algorithme s'avère nécessaire.

Une application des travaux de List porte aussi à croire que la délibération à propos du modèle agrégatif ne peut être exclue. Prenons le cas classique des agrégations incohérentes ${ }^{54}$. Imaginons trois juges qui délibèrent au terme d'un procès ${ }^{55}$. Les juges doivent déterminer si P, si P implique $\mathrm{Q}$, et si Q (la décision finale). Chaque juge respecte les règles de la cohérence individuelle, mais la cohérence collective est laissée au hasard. Voici le résultat auquel arrivent les juges:

Tableau 3: Verdict des différents juges

\begin{tabular}{|l|l|l|l|l|}
\hline & P & P implique Q & Q & Cohérence \\
\hline Juge I & En faveur & En faveur & En faveur & Respectée \\
\hline Juge 2 & En défaveur & En faveur & En défaveur & Respectée \\
\hline Juge 3 & En faveur & En défaveur & En défaveur & Respectée \\
\hline Verdict agrégatif & P & P implique Q & Non-Q & Non respectée \\
\hline
\end{tabular}

52. Ibid., p. 40.

53. Ibid., p. 43.

54. Christian List, "Collective Wisdom. Lessons from the Theory of Judgment Aggregation ", in Hélène Landemore et Jon Elster, dir., Collective Wisdom: Principles and Mechanisms, Cambridge, Cambridge University Press, 20I 2, p. 2 I6-2I 7.

55. Christian List et Philip Pettit, «Aggregating Sets of Judgments: An Impossibility Result », Economics and Philosophy, vol. I8, n I, 2002, p. 92-96. 
Ici, le problème de la cohérence est lié au problème de l'agrégation des attitudes. Toutes les procédures agrégatives n'ont pas le même potentiel épistémique, puisque certaines préservent la cohérence du groupe et pas d'autres. La procédure précédente, en particulier, est trop simple pour préserver la cohérence du groupe. Cela dit, il existe de nombreux modèles préservant la cohérence du groupe, et chaque modèle peut mener à une conclusion différente ${ }^{56}$. L'agrégation touchant des décisions collectives peut donc prendre différentes formes. Si une procédure agrégative cohérente particulière doit être retenue, alors le choix d'une telle procédure doit faire l'objet d'une délibération au sein du groupe.

Nous pouvons tirer des conclusions beaucoup plus fortes de l'argument d'Origgi et de List, notamment quant à la possibilité même de réduire les décisions collectives à un modèle agrégatif. Les systèmes de réputation existants supposent que l'agent comprend le fonctionnement de l'outil qu'il utilise, et qu'il peut faire une évaluation objective de cet outil. Ici, nous sommes face à un double problème. D'une part, peu de gens savent ou disposent des compétences pour comprendre comment les algorithmes de classification des préférences fonctionnent ${ }^{57}$. D'autre part, même si les agents disposaient de ces connaissances, cela ne règle pas le problème. Le succès des algorithmes agrégatifs dépend en partie du fait que l'on ne sait pas comment ces algorithmes fonctionnent. Par exemple, si des informaticiens savent comment fonctionne l'algorithme d'un moteur de recherche, ils peuvent utiliser ces informations pour avantager leur propre site, de manière artificielle, dans le classement des résultats. Tout comme la délibération, un algorithme agrégatif peut donc aussi être victime des relations sociales néfastes qu'il génère. Comme il est possible de détourner les règles des cadres délibératifs, il est tout aussi possible de détourner les règles d'un algorithme.

Au mieux, nous venons de montrer que l'agrégation peut souffrir des mêmes problèmes que la délibération, ou qu'elle ne peut évacuer complètement cette dernière. Si les deux modèles ont un potentiel similaire et souffrent des mêmes problèmes, cela montre simplement que les deux avenues sont similaires. Cette première étape est pertinente mais ne nous permet pas, hors de tout doute, d'écarter l'argument agrégatif. Nous avons besoin d'une preuve plus substantielle du problème de l'agrégation, qui permettrait d'établir clairement la valeur normative supérieure de la délibération. Dans la prochaine section, nous exposons l'analyse conceptuelle d'Andler, qui

56. Par exemple, si l'on considère que les votes sur $\mathrm{P}$, et sur $\mathrm{P}$ implique Q sont suffisants pour inférer Q sans voter sur Q, alors le verdict collectif serait Q. Or, si les juges doivent expliquer leur décision quant à $\mathrm{P}$, et quant à $\mathrm{P}$ implique $\mathrm{Q}$, mais que le seul vote déterminant concerne $\mathrm{Q}$, alors le résultat collectif serait non-Q.

57. Origgi, «Designing Wisdom through the Web: Reputation and the Passion for Ranking", p. 48 . 
montre que le potentiel épistémique d'une agrégation est, de par sa nature même, plus limité qu'une véritable délibération.

\section{Un retour à la délibération: Andler et la distinction entre intelligence et sagesse}

L'argument d'Andler nécessite d'abord d'établir quelques définitions. Andler pose deux formes opposées de processus décisionnels: les processus minces (thin) et les processus denses (thick). Les processus denses sont ceux où la délibération est critique et présente, et où les acteurs communiquent entre eux pour trouver la meilleure solution à un problème. Les processus minces sont ceux où les acteurs mettent simplement les idées qu'ils soutiennent en commun, avec un mécanisme ou un algorithme d'agrégation. La différence majeure entre ces deux processus est dans l'interaction entre les acteurs: dans un processus dense, les interactions entre différents agents (échanges, discussion, consultation, etc.) font partie intégrante de la démarche, alors que c'est le contraire dans un processus mince $^{58}$. Par exemple, un sondage en ligne est un processus mince, alors qu'une commission d'enquête est un processus dense. Andler affirme enfin que la sagesse est "de dégager une conclusion, tout bien considéré » (to draw a conclusion all things considered ${ }^{59}$ ), en incluant différents facteurs comme la cohérence, l'émotion ou l'intelligence.

Le plan de l'argument d'Andler est simple: l'interaction et l'échange au sein des processus denses font office d'occasions éducatives informelles. Bien que le cadre délibératif ne soit pas pensé en fonction d'une mission éducative, il peut donner un sens plus clair et cohérent au projet des agents par l'échange. Que ce soit en comprenant les idées auxquelles ils s'opposent ou en acquérant de nouveaux outils conceptuels, les agents gagnent à interagir avec les autres. Or, pour participer à ces cadres, les agents doivent accepter certaines "responsabilités épistémiques». Un agent ne peut prétendre participer à une occasion éducative informelle si, par exemple, il refuse de changer d'avis lorsqu'il est confronté à des arguments suffisants qui vont à l'encontre de ses convictions. À long terme, le fait, pour un groupe, d'encourager les occasions éducatives informelles, et le respect des responsabilités épistémiques par les différents individus composant le groupe, génère une culture commune caractérisée par la sagesse collective.

58. Daniel Andler, "What Has Collective Wisdom to do with Wisdom?", in Hélène Landemore et Jon Elster, dir., Collective Wisdom: Principles and Mechanisms, Cambridge, Cambridge University Press, 201 2, p. 74.

59. Ibid., p. 83. 


\subsection{Occasions éducatives informelles, responsabilisation des agents et culture commune}

Le fait de participer à une délibération implique de respecter les paramètres épistémiques de cette dernière. Plus précisément, pour que la délibération soit une véritable occasion d'éducation informelle, les agents doivent accepter certaines responsabilités de nature épistémiques. L'idée de responsabilité fait écho au devoir qu'ont les agents de répondre de certaines décisions ou actions. Dans un cadre délibératif, les agents sont responsables s'ils acceptent les décisions collectives motivées, en apparence, par des raisons suffisantes. Par exemple, lorsque, au cours des échanges, les agents en viennent à statuer que $\mathrm{X}$ implique $\mathrm{Y}$, alors un agent ne peut plus accepter $\mathrm{X}$ sans aussi accepter Y. Un agent qui refuserait d'accepter cette implication, même au terme d'une délibération bien menée, ne serait pas responsable. Nous prenons en quelque sorte le "risque» de devoir changer d'avis, sans quoi nous ne sommes pas engagés dans une authentique discussion critique ${ }^{60}$.

Le fait de respecter les paramètres épistémiques de la délibération se vérifie par des dispositions particulières. Un agent qui échange avec d'autres doit être prêt à abandonner ses positions s'il constate qu'un meilleur argument a été identifié. Ou encore, si les raisons à l'origine de la position d'un agent ne résistent pas à un examen critique clair, suivant un fil logique valide, alors l'agent doit être prêt à reconnaître que sa position est injustifiée. De la même manière, mettre fin à une délibération parce que les conclusions qui s'en dégagent nous sont défavorables est une façon de manquer à ses responsabilités. Ainsi, participer à la délibération implique d'accepter les règles constitutives de cette dernière, sans quoi nous manquons à nos responsabilités.

Nothing short of a self can take responsibility for putting an end to deliberation [...] (Epistemic) wisdom is the capacity to guide the epistemic trajectory of an individual $[\ldots]$ and to take responsibility for the individual's final, "all things considered" decisions regarding his beliefs and other epistemic attitudes and dispositions ${ }^{61}$.

S'ils acceptent leurs responsabilités épistémiques, à force d'interagir et de comprendre certaines idées, les agents en viennent à créer une culture commune au sein du groupe. La culture du groupe reflète son expérience partagée, à long terme, au cours de nombreuses délibérations ${ }^{62}$. En effet, si les agents sont responsables, ils ne peuvent faire fi du fait que des conclusions communes se dégagent de la délibération, et doivent donc accepter ces conclusions. La combinaison d'une occasion informelle éducative à la présence de certaines responsabilités épistémiques est donc intimement liée au fait de créer un environnement propice à la formation d'une culture commune.

60. Ibid.

61. Andler, "What Has Collective Wisdom to do with Wisdom? », p. 84-85.

62. Ibid., p. 90-9I. 


\subsection{Les limites internes des processus minces contre le potentiel épistémique des processus denses}

Andler a donc identifié une bonification épistémique propre à la délibération, mais qui se distingue I) de la bonification épistémique que nous retrouvons dans l'argument instrumental en faveur de la démocratie, ou 2) des modèles agrégatifs. Il y a évolution positive du groupe par les échanges et la délibération, mais cette amélioration du groupe ne se limite pas à identifier la meilleure réponse dans un temps donné. La bonification est à trouver dans l'amélioration des agents d'une collectivité, lorsque ceux-ci font des gains épistémiques ${ }^{63}$. C'est tout le contraire dans les processus minces, puisqu'aucune structure ne favorise les occasions informelles d'éducation, la reconnaissance des responsabilités mutuelles et la culture commune. Dans les processus minces, une décision peut donc être intelligente (parce que la meilleure réponse a effectivement été identifiée dans un temps donné), mais elle ne s'accompagne pas de cette bonification épistémique spécifique à la délibération.

Un exemple pourrait faciliter la compréhension de l'argument d'Andler. Supposons qu'une collectivité doive se pencher sur un problème politique urgent. Pour répondre à ce problème, une équipe d'ingénieurs a conçu un modèle inférentiel et agrégatif, alimenté par les réponses des citoyens à un questionnaire. Les citoyens devraient répondre à différentes questions, et au terme de cet exercice, la meilleure décision à prendre serait aisément identifiée. D'un autre côté, une commission d'enquête inviterait les participants à exposer leurs arguments, les faits qu'ils jugent pertinents, et à répondre aux objections adressées à leur endroit. Tous les citoyens ne pourraient pas participer à la commission d'enquête, mais des représentants de différents groupes pourraient s'y prononcer, et les audiences seraient diffusées dans la sphère publique.

Ces deux options reprennent en quelque sorte la distinction entre processus minces et processus denses. Supposons que la collectivité ait seulement le choix entre ces deux options décisionnelles. Si la collectivité opte pour le modèle agrégatif et inférentiel, outre les ingénieurs, personne n'aura véritablement accès aux raisons qui font qu'une décision est la meilleure à prendre. Dans ce contexte, personne ne manque à ses responsabilités en contestant la décision collective, puisque la notion de responsabilité renvoie au fait d'accepter des décisions collectives motivées par des raisons apparentes.

Si la collectivité opte pour la commission d'enquête, elle aura la chance de comprendre pourquoi une décision est préférable à d'autres. Ainsi, le processus dense offre une occasion éducative informelle aux agents composant la collectivité. De plus, si la collectivité refuse les conclusions de la 
commission et ne peut pas invoquer de raisons pour refuser ces conclusions, alors le groupe manquera à ses responsabilités épistémiques. C'est tout le contraire dans le cas d'un processus mince: si les agents ignorent ou désapprouvent publiquement la décision collective mais qu'ils ne comprennent pas les fondements ou les raisons qui motivent cette dernière, ils ne manquent pas à leurs responsabilités épistémiques.

Revenons à l'argument instrumental en faveur de la démocratie. Les processus minces peuvent certes mener une collectivité à prendre la meilleure décision. Or ce n'est pas parce qu'une collectivité est informée de ce qui serait la meilleure décision que la collectivité comprend pourquoi c'est la meilleure décision ${ }^{64}$, et que le fait de prendre cette décision ne mène pas forcément les membres de la collectivité à respecter leurs responsabilités épistémiques ${ }^{65}$. Dans ce contexte, on voit mal pourquoi le groupe agrégatif garantirait les conditions de la sagesse commune. Prise au sens strict, l'affirmation "de dégager une conclusion tout bien considérée ${ }^{66}$ » nécessite d'analyser les différentes dimensions d'une décision, et rien n'assure cette analyse dans les processus minces.

\section{La démocratie délibérative, une question de gains épistémiques ou de légitimité?}

Au terme de l'analyse des processus minces (thin) et denses (thick) chez Andler, il semble effectivement manquer un certain potentiel épistémique aux processus agrégatifs, soit la présence d'occasions informelles éducatives, la responsabilisation mutuelle des agents et la possibilité d'une culture commune. L'interaction entre ces trois aspects de la délibération mène les agents à mieux comprendre le projet auquel ils participent. Andler en conclut que, contrairement aux collectivités régies par des processus denses, les groupes articulés autour de processus minces n'ont pas accès à la "sagesse collective ".

Nous gagnerions toutefois à approfondir la nature de cet argument dans le cas spécifique des décisions politiques. La question de la légitimité n'est pas traitée par Andler ${ }^{67}$. Bien que, suivant l'analyse d'Andler, il semble y avoir une forme particulière de gains épistémiques propres à la délibération, ces gains sont-ils essentiels pour que l'argument soit concluant? En effet, nous pourrions répondre à l'argument d'Andler que les occasions éducatives informelles sont déjà justifiées pour d'autres raisons, notamment quant à la nécessité d'une justification publique des décisions communes, et

64. Ibid., p. 87.

65. Ibid., p. 9 I-92.

66. Ibid., p. 83 .

67. Andler vise à identifier les distinctions constitutives entre processus minces et processus denses. Sa réflexion est donc essentielle pour déterminer ce qui est propre à la délibération. Or nous ne pouvons pas simplement reprendre ses conclusions et les appliquer directement à une réflexion politique sans un examen plus détaillé. 
que nous ne sommes pas obligés d'accepter la valeur épistémique de ces occasions pour les justifier. Pourquoi, dans ce contexte, devrions-nous introduire une nouvelle justification des cadres éducatifs informels, alors qu'une explication concurrente était pleinement valable? Pourrions-nous rejeter l'agrégation sur la base d'un autre argument plus économique, comme une exigence de justification publique des décisions communes? Puisque cet article interroge la place de la délibération dans les cadres politiques légitimes, il importe donc de se pencher sur cet aspect.

Dans les prochains paragraphes, nous allons montrer qu'à partir des prémisses développées par Andler nous pourrions tracer une explication non épistémique quant au rejet des modèles agrégatifs. Cette explication concurrente ferait plutôt appel aux conditions nécessaires à la justification politique des projets collectifs. Ainsi, un cadre politique délibératif serait légitime, mais pour des raisons extrinsèques à la notion de potentiel épistémique. Nous montrerons ensuite que cette seconde voie, bien qu'en apparence distincte, est intimement liée à un argument de nature épistémique. Plus précisément, on ne peut vérifier l'exigence de justification publique sans un cadre qui encourage le potentiel épistémique des agents constituant le groupe. En résumé, le rejet du modèle agrégatif est lié au fait que ce modèle ne respecte pas l'exigence de justification publique, mais, comme nous le verrons, cette exigence de justification publique ne peut être pensée en dehors du potentiel épistémique des cadres délibératifs.

\subsection{Deux explications de la légitimité délibérative: valeur épistémique et justification publique}

Nous pourrions utiliser les éléments développés par Andler en vue d'une conception de la délibération centrée sur la justification publique, où les gains épistémiques seraient secondaires par rapport à la légitimité que véhiculent les décisions communes. Dans cette seconde interprétation, ce qui importe, c'est l'occasion pour les agents d'avoir accès à la justification publique d'un projet commun, parce qu'il s'agirait d'une condition de légitimité des décisions communes ${ }^{68}$. Le principe de base à l'œuvre dans cette approche est qu'on ne peut pas solliciter les agents d'une collectivité pour entériner une décision s'il ne leur est pas possible de la comprendre. Si une personne n'est pas informée des différentes dimensions d'une décision commune, elle n'est sans doute pas informée des raisons qui justifient cette décision, puisque ces deux aspects vont de pair. Ainsi, sans donner accès à des informations et arguments justifiés publiquement, un groupe ne respecte pas les conditions nécessaires pour que ses membres puissent entériner une décision.

68. Amy Gutmann et Dennis Thompson, «Pourquoi la démocratie délibérative est-elle différente?», Philosophiques, vol. 29, n² 2, 2002, p. I94-I95. 
Le lien entre justification publique et cadre éducatif informel est donc saillant. Pour qu'une décision soit légitime, il semble nécessaire d'établir des mécanismes de partage et de justification de cette décision. Ces mécanismes sont une condition de possibilité de la justification publique des décisions communes ${ }^{69}$. Les États qui ne prévoiraient pas ces mécanismes perdraient en légitimité ${ }^{70}$. Lafont expose cette approche dans le passage suivant: "According to this view, public deliberation contributes to democratic legitimacy to the extent that it enables citizens to endorse the laws and policies to which they are subject as their own ${ }^{71}$.» Les cadres éducatifs informels dont Andler fait état seraient alors nécessaires, non pas parce qu'ils s'accompagnent d'une bonification épistémique (à travers la responsabilisation et la culture commune), mais parce que l'absence de ces cadres violerait un principe de légitimité politique. Au mieux, la valeur épistémique de la délibération vient simplement «surdéterminer» les raisons pour lesquelles la délibération est justifiée.

Un partisan d'une justification politique de la délibération pourrait certes accepter que la délibération offre un bon potentiel épistémique. "The more public deliberation meets standards of public-spiritedness, reciprocity, mutual respect, and so on, the more participants in such deliberation are subject to the force of the better argument ${ }^{72}$." Or ces gains spécifiques seraient les effets collatéraux d'un système justifié selon d'autres critères, notamment quant à sa légitimité. Il est bien sûr appréciable que les cadres éducatifs informels mènent les agents à une forme de responsabilisation épistémique, mais l'importance de ces cadres éducatifs provient du fait qu'ils sont une condition de possibilité d'une juste compréhension, pour les agents, du projet auquel ils participent.

En résumé, un partisan de la démocratie épistémique pourrait rejeter le modèle agrégatif parce qu'il ne mène pas les agents à prendre leurs «responsabilités épistémiques ", et n'est pas propice à la formation d'une culture commune porteuse des meilleures décisions collectives, alors qu'un partisan de la légitimité démocratique pourrait rejeter le modèle agrégatif pour une tout autre raison, soit qu'il faut assurer des mécanismes publics de diffusion et de justification des décisions communes. Dans les deux cas, tous peuvent s'entendre sur la nécessité de maintenir des cadres éducatifs informels. Malgré l'importance accordée à ces occasions éducatives, les deux approches ne reconnaissent pas la légitimité des cadres délibératifs pour les mêmes raisons. La tension réside donc dans l'importance que nous accordons au

69. Samuel Freeman, "The Burdens of Public Justification: Constructivism, Contractualism, and Publicity ", Politics, Philosophy \& Economics, vol. 6, n I, 2007, p. I6.

70. Gaus, Justificatory Liberalism, p. I47-I48, 230.

71. Cristina Lafont, «Deliberation, Participation, and Democratic Legitimacy: Should Deliberative Mini-publics Shape Public Policy? ", Journal of Political Philosophy, vol. 23, n I, $20 \mathrm{I} 5$, p. 45 .

72. Ibid. 
fait que, dans un ensemble politique, les agents progressent sur le plan épistémique au fil de la délibération. Il pourrait n'y avoir aucun progrès épistémique des agents composant le groupe, ni aucune culture commune consensuelle qui serait le fruit de ce progrès à long terme, et l'éducation informelle serait néanmoins un aspect nécessaire du régime démocratique. Gaus résume cette distinction dans le passage suivant: "[W]e should be aware that the purpose of public deliberation is not to achieve consensus but to determine what is publicly justified, and the two are not closely tied ${ }^{73}$.»

\subsection{Un potentiel épistémique nécessaire à l'aune du critère de justification publique}

Ce rejet des arguments épistémiques, au profit d'une exigence de justification publique, n'est toutefois pas aussi simple. Nous avons de bonnes raisons de croire qu'il s'agit de deux aspects d'un même processus légitime. Avant de clore cette analyse, nous souhaitons montrer qu'il n'y pas forcément de tension entre, d'une part, l'argument épistémique en faveur de la démocratie délibérative et, d'autre part, l'argument politique axé sur la nécessité d'une justification publique des décisions communes. Nous pouvons en effet expliquer pourquoi un partisan du critère de justification publique ne peut faire l'économie du potentiel épistémique de la délibération. Accepter le critère de justification publique implique de favoriser les cadres de discussion où le potentiel épistémique est supérieur.

Une justification est publique dans deux sens distincts: I) elle est présentée à un public apte à comprendre et juger adéquatement de cette justification; et 2) elle fait appel à des raisons qu'une personne peut comprendre indépendamment d'heuristiques, de prémisses ou de raisons controversées ${ }^{74}$.

Pour vérifier la seconde condition, il importe de présenter une justification potentielle à un groupe diversifié. En effet, pour Gaus, étant donné les ressources épistémiques limitées dont chacun dispose, le fait de présenter une justification à un groupe diversifié permet de "tester " la justification à l'aune d'heuristiques, de méthodes et de faits différents. Gaus avance cette idée dans le passage suivant: "One cannot completely scan one's own system, much less those of others; in one respect, at least, public discourse is a cooperative effort in which others help us explore our own commitments $^{75}$. "Si une décision commune peut, à première vue, paraître publique, il faut néanmoins pousser l'exercice plus loin, et confirmer ce statut public par un examen collectif.

Or, si les agents qui composent le groupe n'assument pas certaines responsabilités épistémiques, il n'y a pas d'intérêt à mettre une justification

73. Gaus, Justificatory Liberalism, p. 23 I.

74. Steven P. Wall, «Public Justification and the Transparency Argument », The Philosophical Quarterly, I996, p. 504; John Rawls, Political Liberalism, New York, Columbia University Press, I996, p. 243.

75. Gaus, Justificatory Liberalism, p. 230. 
"à l'épreuve" auprès d'un groupe diversifié ${ }^{76}$. La justification $\mathrm{X}$ ne gagne pas en légitimité si personne n'a tenté sérieusement de l'invalider à partir d'arguments valables. Cela signifie par conséquent que la légitimité dépend du fait que, pour le bien de la délibération, certaines personnes doivent participer à un exercice critique collectif. C'est précisément parce que les agents composant le groupe font valoir des arguments valides, ne mettent pas fin à la délibération avant que les justifications suffisantes aient passé le test de la critique, et acceptent l'idée même d'un progrès argumentatif, que l'on peut «tester» la validité d'une justification. Il s'avère que tous les aspects précédents (invoquer des arguments valides, clore la délibération seulement sous des conditions spécifiques, accepter le progrès argumentatif) correspondent aux responsabilités épistémiques d'Andler. Et plus un groupe assume adéquatement ses responsabilités épistémiques, plus il est apte à générer des justifications pleinement publiques. Par conséquent, les responsabilités épistémiques sont intimement liées à la notion de justification publique. Le premier aspect est la condition de vérification du second. Un partisan de la justification publique ne peut donc éviter la question des gains épistémiques auprès des agents qui composent le groupe.

En somme, l'obligation de justifier publiquement les décisions qui sont prises pour un groupe implique que celui-ci accepte certaines responsabilités épistémiques. Pour le dire autrement, ce que nous répondons au partisan de la justification publique, c'est qu'il ne peut pas faire l'économie des gains épistémiques auprès des agents composant le groupe. C'est bien parce que les agents assument leurs responsabilités épistémiques qu'il y a vérification du caractère public d'une décision. La délibération est ce qui permet aux agents de comprendre et assumer leurs responsabilités épistémiques, et ces responsabilités épistémiques sont nécessaires à la notion même de justification publique. Les gains épistémiques décrits par Andler et l'exigence de justification publique de Gaus ne sont donc pas deux explications concurrentes de la valeur de la délibération, mais plutôt deux aspects intimement liés de ce qui lui confère une valeur.

\section{Conclusion}

Dans les dernières décennies, de nombreux théoriciens ont justifié le régime démocratique en vertu de ses qualités épistémiques. L'argument est simple: si nous voulons un régime ayant le meilleur potentiel épistémique, alors nous devons adopter un régime de la diversité et de la délibération. Cette justification tombe toutefois dans un piège qu'elle devait initialement désamorcer: si le but ultime que nous visons est l'optimisation du potentiel épistémique, compris comme le fait de «trouver la meilleure décision à prendre dans un temps donné», et que les approches agrégatives atteignent cet

76. Gutmann et Thompson, «Pourquoi la démocratie délibérative est-elle différente? », p. 201. 
objectif, alors nous ne pouvons plus justifier la délibération sur cette base. Le but de cette analyse était moins de défendre la valeur des modèles agrégatifs que de montrer les conséquences logiques controversées d'une argumentation épistémique instrumentale en faveur de la démocratie.

$\mathrm{Au}$ terme de notre parcours, l'agrégation ne semble pas constituer une option décisionnelle valable dans la sphère politique. Cependant, le fait d'abandonner un critère épistémique, comme le fait de prendre la "meilleure décision possible ", au profit d'un critère mixte comprenant à la fois la valeur épistémique d'une décision et l'amélioration épistémique des agents, devait être clarifié. Nous ne pouvions pas nous contenter de juxtaposer l'analyse d'Andler aux cadres politiques sans précisions supplémentaires.

Si l'argument de la meilleure décision a toujours été un trait distinctif des théories épistémiques de la démocratie, la nécessité de diffuser une décision et la justification à l'origine de cette décision n'est pas un trait distinctif de l'argument instrumental. D'autres théories ont justifié la nécessité de cadres éducatifs informels, tout en faisant l'économie des conséquences épistémiques avantageuses de tels cadres. C'est le cas, notamment, de l'exigence de justification publique. Pour résoudre ce problème, nous avons défendu l'idée selon laquelle la justification publique implique la responsabilisation épistémique des membres composant un groupe. La responsabilisation épistémique à travers la délibération n'est donc pas un effet appréciable collatéral de la justification publique: elle est une condition de possibilité de la vérification des justifications publiques. Il s'agit d'une reconfiguration importante du rôle des facteurs épistémiques dans les théories de la démocratie.

En résumé, la raison pour laquelle nous rejetons le modèle agrégatif est qu'il n'implique ni la présence de cadres éducatifs informels ni la responsabilisation épistémique des agents composant le groupe. Pour cette raison, le modèle agrégatif ne respecte pas l'exigence de justification publique. L'exigence de justification publique n'est ici pas étrangère au potentiel épistémique du cadre délibératif. Prendre acte de données appuyées, invoquer des arguments valides, clore la délibération seulement sous des conditions spécifiques, et accepter le progrès argumentatif sont les conditions nécessaires à la fois au progrès épistémique et à la vérification de la justification publique. Il existe donc une connexion marquée entre les théories de la justification publique et les théories de la démocratie épistémique.

\section{Bibliographie}

Andler, Daniel. «What Has Collective Wisdom to do with Wisdom?», in Collective Wisdom: Principles and Mechanisms, sous la direction de Hélène Landemore et Jon Elster, Cambridge, Cambridge University Press, 20I 2, p. 72-94.

Chanu, Frédéric. "Intelligence collective et démocratie en Politique, III, I I ", Ithaque, $\mathrm{n}^{\circ} \mathrm{I} 5,20 \mathrm{I} 4$, p. 75-102.

Dilhac, Marc-Antoine. La tolérance, un risque pour la démocratie? Théorie d'un impératif politique, Paris, Librairie philosophique J. Vrin, 2014. 
Elster, Jon. "L'usage stratégique de l'argumentation", Négociations, vol. 2, n ${ }^{\circ}$, 2005, p. 59-82.

Estlund, David. "Beyond Fairness and Deliberation: the Epistemic Dimension of Democratic Authority", in Deliberative Democracy: Essays on Reason and Politics, sous la direction de James Bohman et William Rheg, Cambridge, MIT Press, I997, p. I73-204.

Freeman, Samuel. "The Burdens of Public Justification: Constructivism, Contractualism, and Publicity ", Politics, Philosophy \& Economics, vol. 6, n I, 2007, p. 5-43.

Gaus, Duluth, et F. Gerald. Justificatory Liberalism: An Essay on Epistemology and Political Theory: An Essay on Epistemology and Political Theory, New Yok, Oxford University Press, USA, I996.

Giroux, Jessy. "Le spectre épistocratique», Philosophiques, vol. 40, n 2, 2013, p. 30I-3I9.

Gutmann, Amy et Dennis Thompson. "Pourquoi la démocratie délibérative est-elle différente?", Philosophiques, vol. 29, n² 2, 2002, p. I93-2 I4.

Hong, Lu et Scott Page. "The Micro-Foundations of Collective Wisdom ", in Collective Wisdom: Principles and Mechanisms, sous la direction de Jon Elster et Hélène Landemore, Cambridge, Cambridge University Press, 20 2, p. 56-7I.

Klein, Mark. "How to Harvest Collective Wisdom on Complex Problems: An Introduction to the MIT Deliberatorium », 20I2, [http://cci.mit.edu/klein/papers/ deliberatorium-intro.pdf].

Lafont, Cristina. «Deliberation, Participation, and Democratic Legitimacy: Should Deliberative Mini-publics Shape Public Policy?", Journal of Political Philosophy, vol. 23, $\mathrm{n}^{\circ} \mathrm{I}, 20 \mathrm{I} 5, \mathrm{p} .40-63$.

Landemore, Hélène. "Pourquoi le grand nombre est plus intelligent que le petit nombre, et pourquoi il faut en tenir compte ", Philosophiques, vol. 40, $\mathrm{n}^{\circ} 2$, 20I3, p. 283-299.

Landemore, Helene E. «Democratic Reason: The Mechanisms of Collective Intelligence in Politics ", in Collective Wisdom: Principles and Mechanisms, sous la direction de Hélène Landemore et Jon Elster, Cambridge, Cambridge University Press, 20I 2, p. 25 I-289.

Le Goff, Alice et Christian Nadeau. "Autorité démocratique et contestation. L'apport d'une approche épistémique ", Philosophiques, vol. 40, n² 2, 20I3, p. $255^{-}$ 28 I.

List, Christian. "Collective Wisdom. Lessons from the Theory of Judgment Aggregation », in Collective Wisdom: Principles and Mechanisms, sous la direction de Hélène Landemore et Jon Elster, Cambridge, Cambridge University Press, 2OI2, p. 203-229.

List, Christian et Philip Pettit. «Aggregating Sets of Judgments: An Impossibility Result ", Economics and Philosophy, vol. I 8, n ${ }^{\circ}$ I, 2002, p. 89-I Io.

Manin, Bernard. "Comment promouvoir la délibération démocratique?", Raisons politiques, vol. 42, $\mathrm{n}^{\circ}$ 2, 20I I, p. 83-II3.

Mansbridge, Jane et al. "The Place of Self-Interest and the Role of Power in Deliberative Democracy*", Journal of Political Philosophy, vol. I8, n I, 2010, p. 64-I00.

McCormick, John P. «Machiavelli Against Republicanism On the Cambridge School's "Guicciardinian Moments" ", Political Theory, vol. 3 I, nº 5, 2003, p. 6I 5-643. 
Mouffe, Chantal. "Le politique et la dynamique des passions ", Politique et sociétés, vol. 22, $\mathrm{n}^{\circ} 3,2003$, p. I43-I 54 .

Origgi, Gloria. «Designing Wisdom through the Web: Reputation and the Passion for Ranking", in Collective Wisdom: Principles and Mechanisms, sous la direction de Hélène Landemore et Jon Elster Cambridge, Cambridge University Press, 20I2, p. 38-55.

Page, Scott E. Diversity and complexity, Princeton (NJ), Princeton University Press, 2010.

- The Difference: How the Power of Diversity Creates Better Groups, Firms, Schools, and Societies, Princeton (NJ), Princeton University Press, 2007.

Rawls, John. Political Liberalism, New York, Columbia University Press, I 996.

Roussin, Juliette. "Démocratie contestataire ou contestation de la démocratie? L'impératif de la bonne décision et ses ambiguïtés ", Philosophiques, vol. 40, $\mathrm{n}^{\circ} 2$, 20I3, p. 369-397.

Roussin, Juliette, "Sagesse des foules ", La vie des idées, 2 I septembre 20 I $2,<$ http:// www.laviedesidees.fr/Sagesse-des-foules.html>,

Sanders, Lynn M., «Against Deliberation ", Political Theory, vol. 25, n 3, I997, pp. 347-376.

Schkade, David, Sunstein, Cass R. et Hastie, Reid, "What Happened on Deliberation Day?", ID 9ıг646, Rochester, NY, Social Science Research Network, 2006, <http://dx.doi.org/I0.2 I39/ssrn.91 I 646>,.

Servan-Schreiber, Emile, "Trading Uncertainty for Collective Wisdom ", in Landemore, Hélène et Elster, Jon, dir., Collective Wisdom: Principles and Mechanisms, Cambridge, Cambridge University Press, 20I 2, pp. 2 I-37.

Solomon, Miriam, "Groupthink versus The Wisdom of Crowds: The Social Epistemology of Deliberation and Dissent ", The Southern Journal of Philosophy, vol. 44, no. Si, 2006, pp. 28-42.

Sunstein, Cass R., Infotopia: How Many Minds Produce Knowledge, Oxford University Press, 2006.

Surowiecki, James, The wisdom of crowds, New York, Anchor, 2005.

Urbinati, Nadia, Democracy Disfigured, Harvard University Press, 20 I4.

Vermeule, Adrian, "Collective Wisdom and Institutional Design ", in Landemore, Hélène et Elster, Jon, dir., Collective Wisdom: Principles and Mechanisms, Cambridge, Cambridge University Press, 201 2, pp. 338-367.

Wall, Steven P. «Public Justification and the Transparency Argument », The Philosophical Quarterly, I996, p. 50I-507. 